\title{
Non-Newtonian fluid flow in annular pipes and entropy generation: Temperature-dependent viscosity
}

\author{
M YÜRÜSOY ${ }^{a}$, B S YILBAŞ $^{b *}$ and M PAKDEMIRLI ${ }^{b}$ \\ ${ }^{a}$ Technical Education Faculty, Afyon Kocatepe University, Afyon, Turkey \\ ${ }^{b}$ Department of Mechanical Engineering, King Fahd University of Petroleum and \\ Minerals, Dhahran 31261, Saudi Arabia \\ *e-mail: bsyilbas@kfupm.edu.sa
}

MS received 26 May 2005; revised 27 March 2006

\begin{abstract}
Non-Newtonian fluid flow in annular pipes is considered and the entropy generation due to fluid friction and heat transfer in them is formulated. A third-grade fluid is employed to account for the non-Newtonian effect, while the Reynolds model is accommodated for temperature-dependent viscosity. Closed-form solutions for velocity, temperature, and entropy fields are presented. It is found that entropy generation number increases with reducing non-Newtonian parameter, while it is the reverse for the viscosity parameter, which is more pronounced in the region close to the annular pipe inner wall.
\end{abstract}

Keywords. Non-Newtonian fluid; third-grade fluid; variable viscosity; entropy; entropy generation number.

\section{Introduction}

Flow through annular pipes finds application in the process industry. In some cases, the fluid may consist of two phases such as coal particles-water mixture (coal slurries) and the numerical modelling of such flow situations is difficult to solve for the conservation equations for each phase and coupled equations for all phases. This requires extensive computational time and effort. However, the fluid can be considered as a homogenous medium with non-Newtonian behaviour. In this case, an analytical solution for the problem may be possible. The solution facilitates correlation of the flow characteristics with the fluid properties.

Considerable research studies were carried out to examine non-Newtonian flow characteristics. The effects of variable viscosity and viscous dissipation on the flow of a third-grade fluid in a pipe were investigated by Massoudi \& Christie (1995). They calculated numerically the velocity and temperature distributions in the pipe. Rajagopal \& Sciubba (1984) studied

*For correspondence

A list of symbols is given at the end of the paper 
the flow of a third-grade non-Newtonian fluid between horizontally situated and heated parallel plates. They employed temperature-dependent viscosity in the analysis. An approximate analytical solution for the flow of third-grade non-Newtonian fluid in a pipe was presented by Yurusoy \& Pakdemirli (2002). They showed that the closed form solutions obtained for velocity and temperature distributions agreed well with the previously obtained numerical results, if certain validity criteria were met. Annular flow for the same problem was further studied by Yurusoy (2004).

The effect of viscosity models on the stability of a non-Newtonian fluid in a channel with heat transfer was examined by Pinarbasi \& Ozalp (2001). They showed that the fluids obeying the Arrhenius law were more stable than those obeying the Nahme law if both models were used on the same temperature-sensitive viscosity, reference viscosity and temperature. The power-law fluid flow in pipes was considered by Hecht (1973). He obtained the general solution to the enthalpy profiles through integrating momentum and energy equations across the pipe cross-section under constant wall-flux conditions. The non-Newtonian fluid parameter estimation using conical flows was considered by Tanner (1966). He indicated that narrow cone angles should be used to measure the effect of the third invariant on the viscosity function. The unsteady axial Couette flow of power-law fluids in concentric annulii was investigated by Wang \& Chukwu (1996). They presented the pressure gradient equations in dimensionless form for different pipe/bore hole diameter ratios and power-law index values.

Entropy generation in the flow systems is due to fluid friction and heat transfer. Moreover, entropy analysis provides information for the quantification of thermodynamic irreversibility in the flow system. Consequently, entropy minimization lowers the frictional and heat transfer losses in the system. Considerable research studies were carried out to examine entropy generation in thermal systems. Bejan (1995) examined the entropy generation and minimization in thermal systems. He indicated that entropy minimization could be used as an effective tool for designing thermal systems. The non-isothermal flow of visco-elastic materials using differential constitutive equations was investigated by Peters \& Baaijens (1997). They examined the partitioning between dissipated and elastically stored energy and discussed the difference between entropy and entropy elasticity. The convective heat transfer in an annular packed bed was investigated by Demirel \& Kahraman (2000). They indicated that the volumetric entropy generation map could be used to identify the excessive entropy generation due to operating conditions or design parameters for a required task. The Shannon entropy characteristics of two-phase flow systems were examined by Zhang \& Shi (1999). They found that the entropy generation of the bubble flow was the smallest, entropy generation in slug flows was the largest, and entropy generation of annular flow was between that of bubble flow and slug flow.

In the present study, non-Newtonian fluid flow in annular pipes is considered and entropy generation due to fluid friction and heat transfer is formulated. A third-grade fluid is employed to account for the non-Newtonian effect, while Reynolds model is accommodated to account for the temperature effect on the viscosity.

\section{Velocity and temperature profiles}

Consider non-Newtonian fluid flow through an annular pipe as shown in figure 1. The nondimensional form of the equations of motion of a third-grade fluid in a pipe with heat transfer was derived by Massoudi \& Christie (1995), which are: 

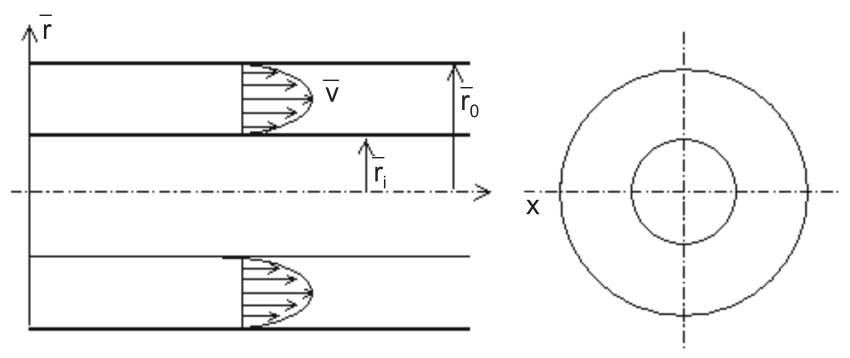

Figure 1. Schematic view of annular pipe and flow situation.

$$
\begin{array}{r}
\frac{\mathrm{d} \mu}{\mathrm{d} r} \frac{\mathrm{d} v}{\mathrm{~d} r}+\frac{\mu}{r}\left(\frac{\mathrm{d} v}{\mathrm{~d} r}+r \frac{\mathrm{d}^{2} v}{\mathrm{~d} r^{2}}\right)+\frac{\Lambda}{r}\left(\frac{\mathrm{d} v}{\mathrm{~d} r}\right)^{2}\left(\frac{\mathrm{d} v}{\mathrm{~d} r}+3 r \frac{\mathrm{d}^{2} v}{\mathrm{~d} r^{2}}\right)=C, \\
\frac{\mathrm{d}^{2} \theta}{\mathrm{d} r^{2}}+\frac{1}{r} \frac{\mathrm{d} \theta}{\mathrm{d} r}+\Gamma\left(\frac{\mathrm{d} v}{\mathrm{~d} r}\right)^{2}\left[\mu+\Lambda\left(\frac{\mathrm{d} v}{\mathrm{~d} r}\right)^{2}\right]=0, \\
v\left(r_{i}\right)=v\left(r_{o}\right)=\theta\left(r_{i}\right)=\theta\left(r_{o}\right)=0 .
\end{array}
$$

The terms are related to the dimensional ones (with overbars) through the following relations,

$$
\begin{gathered}
R^{*}=\bar{r}_{o}-\bar{r}_{i}, \quad R=\frac{\bar{r}_{i}}{\bar{r}_{o}}, \quad r_{i}=\frac{3 R}{1-R}, \quad r_{o}=\frac{3}{1-R}, \\
r=\frac{\bar{r}}{R^{*}}, \quad v=\frac{\bar{v}}{V_{0}}, \quad \theta=\frac{\bar{\theta}-\theta_{w}}{\bar{\theta}_{m}-\theta_{w}}, \quad \mu=\frac{\bar{\mu}}{\mu_{*}} .
\end{gathered}
$$

The dimensionless parameters involved in (1) and (2) are

$$
C=\frac{C_{1} R^{* 2}}{\mu_{*} V_{0}}, \quad C_{1}=\frac{\partial p}{\partial z}, \quad \Gamma=\frac{\mu_{*} V_{0}^{2}}{k\left(\bar{\theta}_{m}-\theta_{w}\right)}, \quad \Lambda=\frac{2 \beta_{3} V_{0}^{2}}{\mu_{*} R^{* 2}} .
$$

If Reynold's model is assumed for the variation of viscosity with temperature,

$$
\mu=\exp (-M \theta)
$$

approximate solutions can be obtained by selecting $\Lambda=\in \lambda$ and $M=\in m$, where $\in$ the perturbation parameter, is a small quantity. The velocity and temperature profiles are (Yurusoy 2004),

$$
\begin{aligned}
v= & \left(C r^{2} / 4\right)+c_{1} \ln (r)+c_{2}+\in\left(\left(t_{0} / r^{2}\right)+t_{1} r^{2}-t_{2} r^{4}-t_{3} r^{6}+t_{4} r^{2} \ln (r)\right. \\
& \left.+t_{5} \ln (r)^{2}-t_{6} r^{2} \ln (r)^{2}-t_{7} \ln (r)^{3}+h_{1} \ln (r)+h_{2}\right), \\
\theta= & -\Gamma\left(\left(C^{2} r^{4} / 64\right)+\left(C c_{1} r^{2} / 4\right)+\left(c_{1}^{2} \ln (r)^{2} / 2\right)\right)+d_{1} \ln (r)+d_{2} \\
& +\in\left(k_{1} r^{8}+k_{2} r^{6}+r^{4}\left(k_{3} \ln (r)^{2}+k_{4} \ln (r)+k_{5}\right)\right. \\
& +r^{2}\left(k_{6} \ln (r)^{2}+k_{7} \ln (r)+k_{8}\right)+k_{9} \ln (r)^{4} \\
& \left.+k_{10} \ln (r)^{3}+k_{11} \ln (r)^{2}+\left(k_{12} / r^{2}\right)+m_{1} \ln (r)+m_{2}\right),
\end{aligned}
$$


where $c_{1}, c_{2}, d_{1}, d_{2}, t_{0,1,2 \ldots 7}, h_{1}, h_{2}, k_{1,2 \ldots, 12}, m_{1}$ and $m_{2}$ are defined as follows,

$$
\begin{aligned}
c_{1}= & {\left[C\left(r_{i}^{2}-r_{o}^{2}\right)\right] / 4 \ln \left(r_{o} / r_{i}\right), \quad c_{2}=-(C / 4)\left(r_{i}^{2}\right)-c_{1} \ln \left(r_{i}\right), } \\
d_{1}= & -\left[\Gamma / \ln \left(r_{o} / r_{i}\right)\right]\left(\left(C^{2}\left(r_{i}^{4}-r_{o}^{4}\right) / 64\right)+\left(C c_{1}\left(r_{i}^{2}-r_{o}^{2}\right) / 4\right)\right. \\
& \left.+\left(c_{1}^{2} / 2\right)\left(\ln \left(r_{i}\right)^{2}-\ln \left(r_{0}\right)^{2}\right)\right), \\
d_{2}= & \Gamma\left(\left[C^{2} / 64\right] r_{i}^{4}+\left(C c_{1} / 4\right) r_{i}^{2}+\left(c_{1}^{2} / 2\right) \ln \left(r_{i}\right)^{2}\right)-d_{1} \ln \left(r_{i}\right), \\
t_{0}= & c_{1}^{3} \lambda / 2, \\
t_{1}= & C\left(-6 C c_{1} \lambda-2 d_{1} m+4 d_{2} m-3 c_{1}^{2} \Gamma m\right) / 16, \\
t_{2}= & C^{2}\left(8 C \lambda+9 c_{1} \Gamma m\right) / 256, \\
t_{3}= & C^{3} \Gamma m / 768, \\
t_{4}= & C m\left(2 d_{1}+c_{1}^{2} \Gamma\right) / 8, \\
t_{5}= & c_{1} d_{1} m / 2, \\
t_{6}= & C c_{1}^{2} \Gamma m / 8, \\
t_{7}= & c_{1}^{3} \Gamma m / 6, \\
h_{1}= & {\left[1 / \ln \left(r_{o} / r_{i}\right)\right]\left[t_{0}\left(\left(1 / r_{i}^{2}\right)-\left(1 / r_{o}^{2}\right)\right)+t_{1}\left(r_{i}^{2}-r_{o}^{2}\right)-t_{2}\left(r_{i}^{4}-r_{o}^{4}\right)\right.} \\
& -t_{3}\left(r_{i}^{6}-r_{o}^{6}\right)+t_{4}\left(r_{i}^{2} \ln \left(r_{i}\right)-r_{o}^{2} \ln \left(r_{o}\right)\right)+t_{5}\left(\ln \left(r_{i}\right)^{2}-\ln \left(r_{o}\right)^{2}\right) \\
& \left.-t_{6}\left(r_{i}^{2} \ln \left(r_{i}\right)^{2}-r_{o}^{2} \ln \left(r_{o}\right)^{2}\right)-t_{7}\left(\ln \left(r_{i}\right)^{3}-\ln \left(r_{o}\right)^{3}\right)\right], \\
& -\Gamma C t_{1} / 8-5 m \Gamma^{2} C^{2} c_{1}^{2} / 256+\Gamma c_{1} t_{2} / 2, \\
k_{6}= & -m \Gamma^{2} C c_{1}^{3} / 8+\Gamma c_{1} t_{6}+3 \Gamma C t_{2} / 4, \\
k_{7}= & m \Gamma^{2} C c_{1}^{3} / 4-\Gamma c_{1} t_{4}-\Gamma C t_{5} / 2-\Gamma c_{1} t_{6}-3 \Gamma C t_{7} / 2+m \Gamma d_{1} c_{1} C / 4, \\
h_{2}= & {\left[\left(-t_{o} / r_{i}^{2}\right)-t_{1} r_{i}^{2}+t_{2} r_{i}^{4}+t_{3} r_{i}^{6}-t_{4} r_{i}^{2} \ln \left(r_{i}\right)\right.} \\
& \left.-t_{5} \ln \left(r_{i}\right)^{2}+t_{6} r_{i}^{2} \ln \left(r_{i}\right)^{2}+t_{7} \ln \left(r_{i}\right)^{3}-h_{1} \ln \left(r_{i}\right)\right], \\
k_{1}= & -m \Gamma^{2} C^{4} / 16384+3 \Gamma C t_{3} / 32, \\
k_{2}= & -\lambda \Gamma C^{4} / 576-5 m \Gamma^{2} C^{3} c_{1} / 2304+\Gamma c_{1} t_{3} / 3+\Gamma C t_{2} / 9, \\
k_{3}= & -m \Gamma^{2} C^{2} c_{1}^{2} / 128+\Gamma C t_{6} / 8, \\
k_{4}= & m \Gamma^{2} C^{2} c_{1}^{2} / 128+m \Gamma d_{1} C^{2} / 64-\Gamma C t_{4} / 8, \\
k_{5}= & -\lambda \Gamma C^{3} c_{1} / 32+m \Gamma d_{2} C^{2} / 64-\Gamma C t_{6} / 64-m \Gamma d_{1} C^{2} / 128 \\
&
\end{aligned}
$$




$$
\begin{aligned}
k_{8}= & \Gamma C t_{5} / 2-\Gamma c_{1} t_{1}-3 \lambda \Gamma c_{1}^{2} C^{2} / 8+m \Gamma d_{2} C c_{1} / 4-\Gamma C k_{1} / 4+\Gamma c_{1} t_{6} / 2 \\
& +\Gamma c_{1} t_{4} / 2-m \Gamma^{2} C c_{1}^{3} / 4-m \Gamma c_{1} C d_{1} / 4+9 \Gamma C t_{7} / 8 \\
k_{9}= & \Gamma c_{1} t_{7} / 2-m \Gamma^{2} c_{1}^{4} / 24 \\
k_{10}= & -2 \Gamma c_{1} t_{5} / 3+m c_{1}^{2} \Gamma d_{1} / 6 \\
k_{11}= & m \Gamma d_{2} c_{1}^{2} / 2-\Gamma c_{1} k_{1}+\Gamma C t_{0}-\lambda \Gamma c_{1}^{3} C \\
k_{12}= & -\lambda \Gamma c_{1}^{4}+4 \Gamma c_{1} t_{0} / 4 \\
m_{1}= & {\left[1 / \ln \left(r_{o} / r_{i}\right)\right]\left[k_{1}\left(r_{i}^{8}-r_{o}^{8}\right)+k_{2}\left(r_{i}^{6}-r_{o}^{6}\right)+\left(r_{i}^{4}-r_{o}^{4}\right)\left(k_{3}\left(\ln \left(r_{i}\right)^{2}-\ln \left(r_{o}\right)^{2}\right)\right.\right.} \\
& \left.+k_{4}\left(\ln \left(r_{i}\right)-\ln \left(r_{0}\right)\right)+k_{5}\right)+\left(r_{i}^{2}-r_{o}^{2}\right)\left(k_{6}\left(\ln \left(r_{i}\right)^{2}-\ln \left(r_{o}\right)^{2}\right)+k_{7}\left(\ln \left(r_{i}\right)\right.\right. \\
& \left.\left.-\ln \left(r_{o}\right)\right)+k_{8}\right)+k_{9}\left(\ln \left(r_{i}\right)^{4}-\ln \left(r_{o}\right)^{4}\right)+k_{10}\left(\ln \left(r_{i}\right)^{3}-\ln \left(r_{o}\right)^{3}\right) \\
& \left.+k_{11}\left(\ln \left(r_{i}\right)^{2}-\ln \left(r_{o}\right)^{2}\right)+k_{12}\left(\left(1 / r_{i}^{2}\right)-\left(1 / r_{o}^{2}\right)\right)\right] \\
m_{2}= & -\left[k_{1} r_{i}^{8}+k_{2} r_{i}^{6}+r_{i}^{4}\left(k_{3} \ln \left(r_{i}\right)^{2}+k_{4} \ln \left(r_{i}\right)+k_{5}\right)+r_{i}^{2}\left(k_{6} \ln \left(r_{i}\right)^{2}\right.\right. \\
& \left.\left.+k_{7} \ln \left(r_{i}\right)+k_{8}\right)+k_{9} \ln \left(r_{i}\right)^{4}+k_{10} \ln \left(r_{i}\right)^{3}+k_{11} \ln \left(r_{i}\right)^{2}+k_{12} / r_{i}^{2}\right] \\
& -m_{1} \ln \left(r_{i}\right) .
\end{aligned}
$$

For $m=0$, solutions reduced to those of the constant viscosity case.

\section{Viscous dissipation and entropy generation}

The dimensional viscous dissipation term $(\bar{\phi})$ can be obtained from equations of motion, i.e.:

$$
\bar{\phi}=\bar{\mu}(\mathrm{d} \bar{v} / \mathrm{d} \bar{r})^{2}+2 \beta_{3}(\mathrm{~d} \bar{v} / \mathrm{d} \bar{r})^{4},
$$

or inserting the dimensionless quantities yields,

$$
\bar{\phi}=\left(\mu_{*} V_{0}^{2} / R^{* 2}\right)(\mathrm{d} v / \mathrm{d} r)^{2}\left[\mu+\Lambda(\mathrm{d} v / \mathrm{d} r)^{2}\right] .
$$

The dimensional volumetric entropy generation is defined as (Bejan 1995):

$$
S_{\text {gen }}^{\prime \prime \prime}=\left(k / \bar{\theta}^{2}\right)(\mathrm{d} \bar{\theta} / \mathrm{d} \bar{r})^{2}+\bar{\phi} / \bar{\theta} .
$$

The first term in (38) is the volumetric entropy generation due to heat transfer and the second term is the entropy generation due to viscous dissipation. Substituting (37) in (38), and expressing the terms in dimensionless forms, we finally obtain:

$$
N s=\frac{1}{\left(\theta+\theta_{0}\right)^{2}}\left(\frac{\mathrm{d} \theta}{\mathrm{d} r}\right)^{2}+\Gamma \frac{1}{\left(\theta+\theta_{0}\right)}\left(\frac{\mathrm{d} v}{\mathrm{~d} r}\right)^{2}\left[\mu+\Lambda\left(\frac{\mathrm{d} v}{\mathrm{~d} r}\right)^{2}\right],
$$


where $N s$ is the entropy generation number. It is defined by dividing the dimensional volumetric entropy generation to a reference volumetric entropy generation $S_{G}^{\prime \prime \prime}$. The relevant definitions are:

$$
N s=S_{\text {gen }}^{\prime \prime \prime} / S_{G}^{\prime \prime \prime}, \quad S_{G}^{\prime \prime \prime}=k / R^{* 2}, \quad \theta=\theta_{w} /\left(\theta_{m}-\theta_{w}\right) .
$$

In (39), the first term due to heat generation can be assigned as $N s_{1}$ and the second term due to viscous dissipation as $\mathrm{Ns}_{2}$, i.e.:

$$
N s_{1}=\left[1 /\left(\theta+\theta_{0}\right)^{2}\right](\mathrm{d} \theta / \mathrm{d} r)^{2}, \quad N s_{2}=\Gamma\left[1 /\left(\theta+\theta_{0}\right)\right](\mathrm{d} v / \mathrm{d} r)^{2}\left[\mu+\Lambda(\mathrm{d} v / \mathrm{d} r)^{2}\right] .
$$

Assuming Reynold's model as given in (6), following Yurusoy (2004), the viscosity can be approximated as $\mu \cong 1-M \theta$. Since the temperature and velocity profiles are known functions, they can be inserted from (7) and (8) into (39) and (41) for final evaluations of the entropy generation numbers. The two parts of the entropy generation number are then,

$$
\begin{aligned}
N s_{1}= & \frac{1}{\left(\theta+\theta_{0}\right)^{2}}\left[-\Gamma\left(\frac{C^{2} r^{3}}{16}+\frac{C c_{1} r}{2}+\frac{c_{1}^{2} \ln (r)}{r}\right)+\frac{d_{1}}{r}\right. \\
& +\in\left(8 k_{1} r^{7}+6 k_{2} r^{5}+r^{4}\left(\frac{2 k_{3} \ln (r)}{r}+\frac{k_{4}}{r}\right)\right. \\
& +4 r^{3}\left(k_{3} \ln (r)^{2}+k_{4} \ln (r)+k_{5}\right)+2 r\left(k_{6} \ln (r)^{2}+k_{7} \ln (r)+k_{8}\right) \\
& +r^{2}\left(\frac{2 k_{6} \ln (r)}{r}+\frac{k_{7}}{r}\right)+\frac{4 k_{9} \ln (r)^{3}}{r}+\frac{3 k_{10} \ln (r)^{2}}{r}+\frac{2 k_{11} \ln (r)}{r} \\
& \left.\left.-\frac{2 k_{12}}{r^{3}}+\frac{m_{1}}{r}\right)\right]^{2}, \\
N s_{2}= & \Gamma \frac{1}{\left(\theta+\theta_{0}\right)}\left[\frac{C r}{2}+\frac{c_{1}}{2}\right. \\
& +\in\left(-\frac{2 t_{0}}{r^{3}}+2 t_{1} r-4 t_{2} r^{3}-6 t_{3} r^{5}+t_{4}(2 r \ln (r)+r)+\frac{2 t_{5} \ln (r)}{r}\right. \\
& \left.\left.-2 t_{6}\left(r \ln (r)^{2}-r \ln (r)\right)-\frac{3 t_{7} \ln (r)^{2}}{r}+\frac{h_{1}}{r}\right)\right]^{2} \\
& \times\left[\mu+\Lambda\left(\frac{C r}{2}+\frac{c_{1}}{2}+\in\left(-\frac{2 t_{0}}{r^{3}}+2 t_{1} r-4 t_{2} r^{3}-6 t_{3} r^{5}\right.\right.\right. \\
& +t_{4}(2 r \ln (r)+r)+\frac{2 t_{5} \ln (r)}{r}-2 t_{6}\left(r \ln (r)^{2}-r \ln (r)\right) \\
& \left.\left.\left.-\frac{3 t_{7} \ln (r)^{2}}{r}+\frac{h_{1}}{r}\right)\right)^{2}\right] \cdot
\end{aligned}
$$

Equations (42) and (43) are used to compute the entropy generation numbers. 

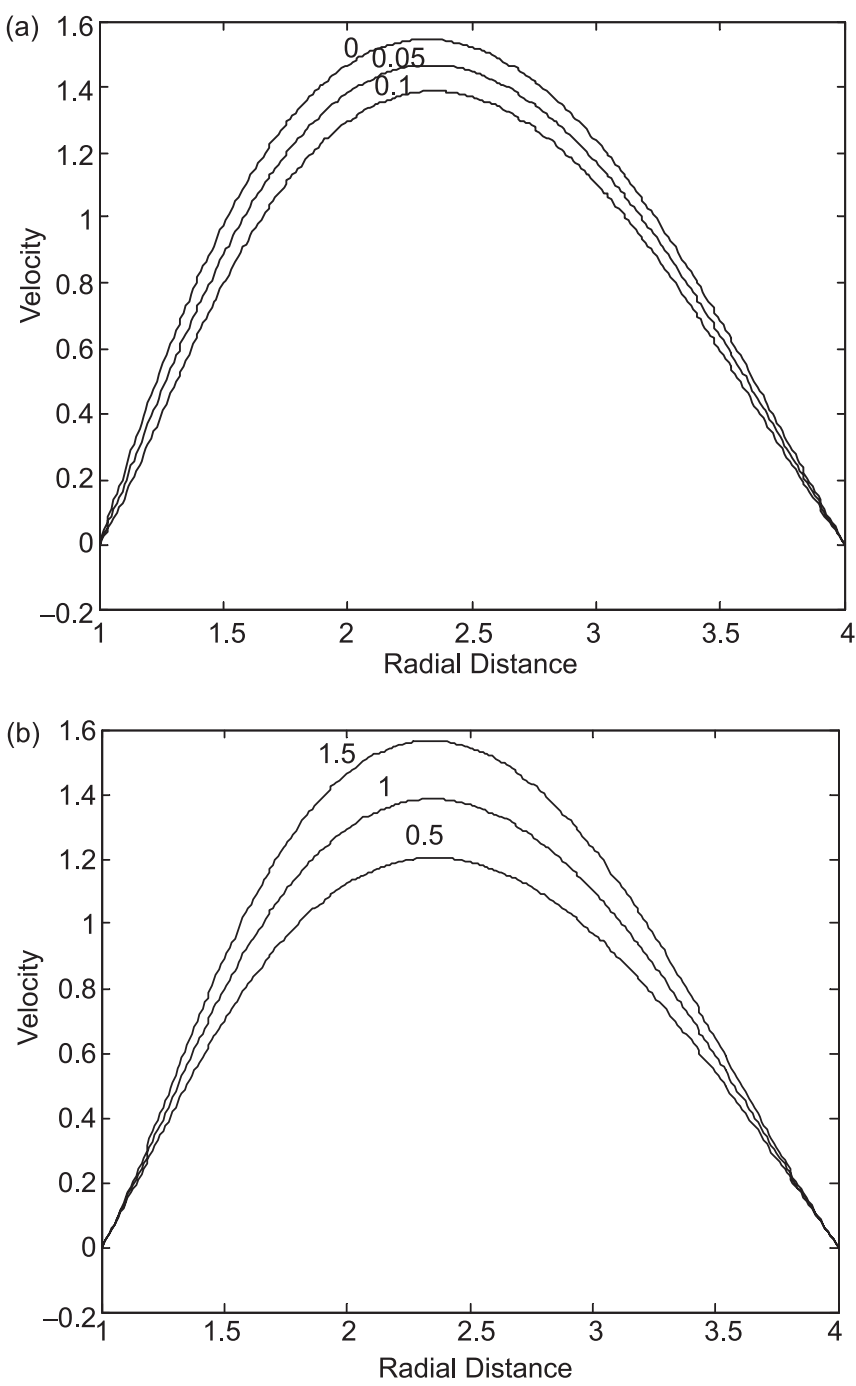

Figure 2. Velocity profiles along the pipe radius for (a) different non-Newtonian parameters $(\Lambda)$ : $C=-1, \Gamma=1$ and $M=1$, and (b) different viscosity parameters (M) $: C=-1, \Gamma=1$ and $\Lambda=$ $0 \cdot 1$.

\section{Results and discussions}

Non-Newtonian fluid flow in an annular pipe is considered. Temperature-dependent viscosity is accommodated while a third-grade fluid is introduced to account for the non-Newtonian effects.

Figures $2 \mathrm{a}$ and $\mathrm{b}$ show velocity profiles along the radial distance in annular pipes for different non-Newtonian parameters and viscosity coefficients respectively. The maximum velocity magnitude increases with reducing non-Newtonian parameter (figure $2 a$ ). In this case, the rate of fluid strain in the region of the annular pipe wall increases. It should be noted that the nonNewtonian parameter is inversely proportional to the fluid viscosity $\left(\Lambda=2 \beta_{3} V_{0}^{2} / \mu_{*} R^{* 2}\right.$ ) with a non-Newtonian parameter of zero magnitude corresponding to a Newtonian fluid. Consequently, a Newtonian fluid results in the highest rate of fluid strain close to the annular pipe wall. In the case of figure $2 b$, the magnitude of velocity increases with the viscosity 

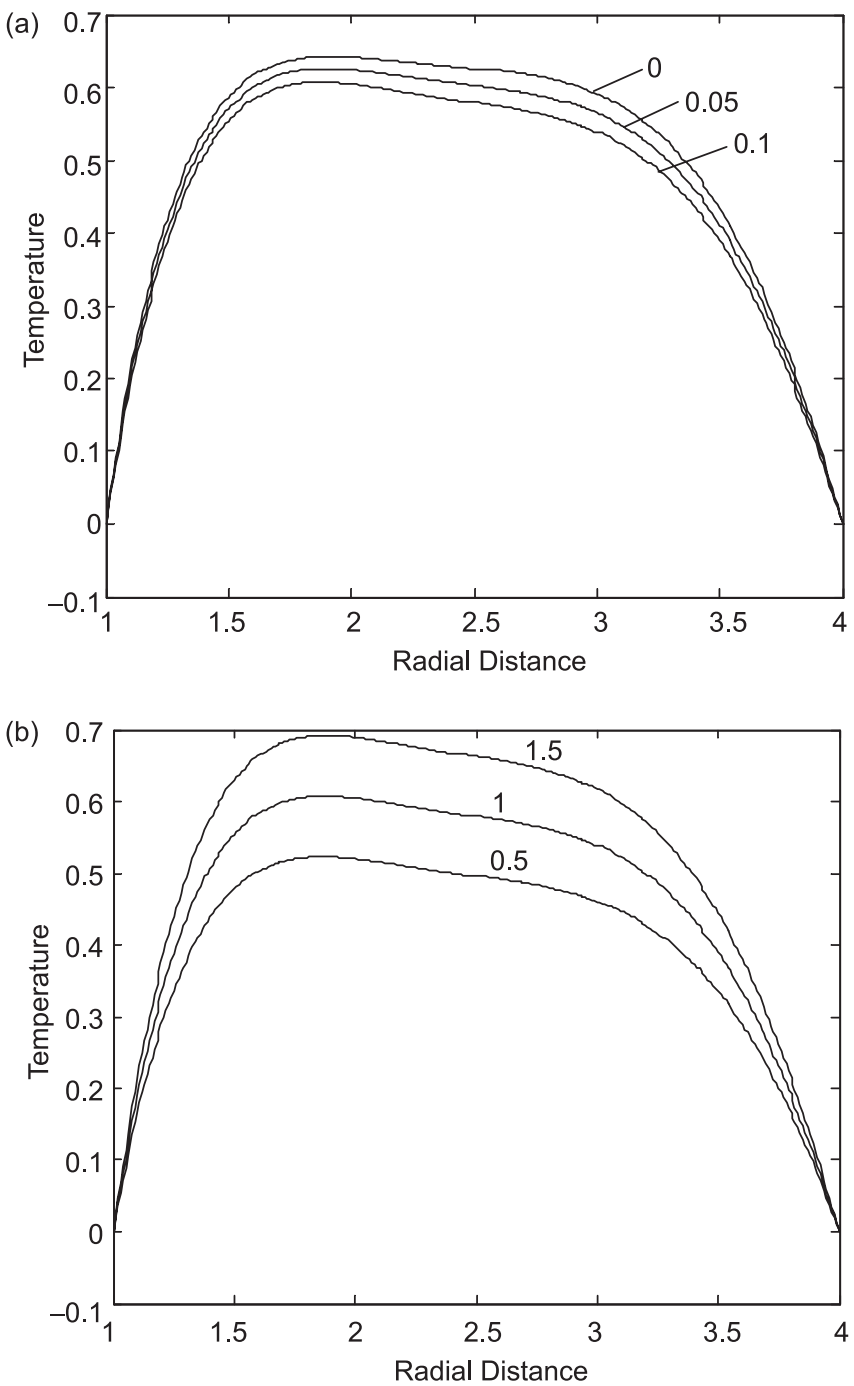

Figure 3. Temperature profiles along the pipe radius for (a) different non-Newtonian parameters $(\Lambda): C=-1, \Gamma=1$ and $M=1$, and (b) for different viscosity parameters $(M): C=$ $-1, \Gamma=1$ and $\Lambda=0 \cdot 1$.

parameter, i.e. reducing viscosity with increasing viscosity parameter enhances the rate of fluid strain near the annular pipe wall and increases maximum velocity magnitude in the pipe.

Figures $3 \mathrm{a}$ and $\mathrm{b}$ show temperature distribution in the annular pipe for different nonNewtonian and viscosity parameters respectively. The maximum temperature increases with reducing non-Newtonian parameter (figure 3a). The convective acceleration of the fluid enhances the heat transfer rates due to convection and conduction effects in the fluid. This, in turn, results in increased fluid temperature. Temperature gradient in the region close to the annular pipe wall increases with reducing non-Newtonian parameter. In the case of figure $3 \mathrm{~b}$, viscosity parameter significantly influences temperature distribution in the annular pipe. Increasing viscosity parameter enhances temperature rise in the fluid. Since viscosity reduces with increasing temperature, convective acceleration improves heat transfer rates in the annular pipe. 

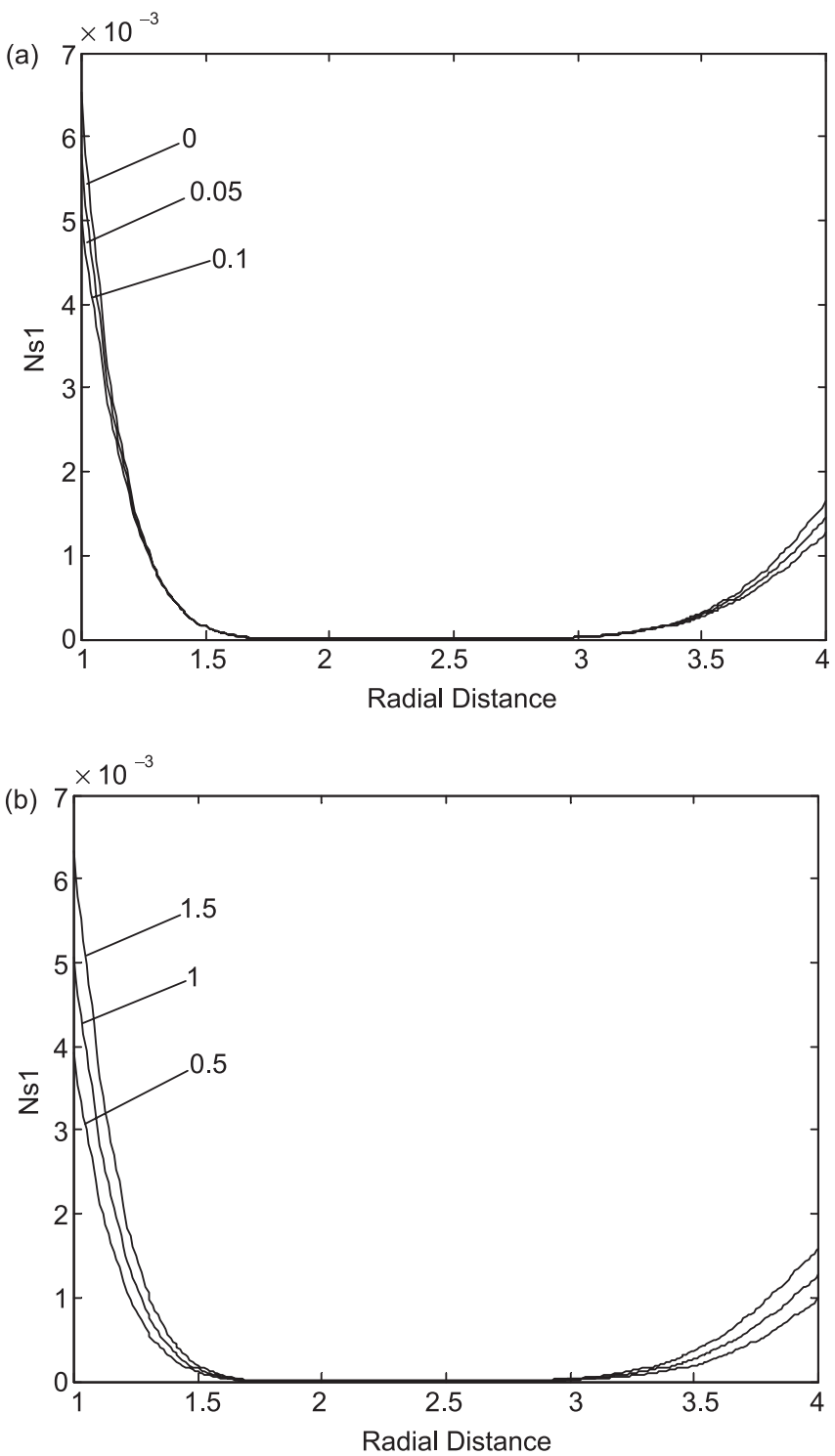

Figure 4. Entropy generation number due to heat transfer along the pipe radius for (a) different non-Newtonian parameters $(\Lambda)$ : $C=-1, \Gamma=1, M=1$ and $\theta_{0}=30$, and (b) different viscosity parameters $(M): C=$ $-1, \Gamma=1, \Lambda=0 \cdot 1$ and $\theta_{0}=$ 30.

Figures $4 \mathrm{a}$ and $\mathrm{b}$ show entropy generation number due to heat transfer for different nonNewtonian and viscosity parameters respectively. Entropy generation number attains high values in the vicinity of the annular pipe wall, which is more pronounced in the region close to the inner wall of the pipe (figure 4a). In this case, high rate of heat transfer in this region enhances the entropy generation rate. Reducing non-Newtonian parameter increases entropy generation rate, in which case the rate of heat transfer increases. In the case of figure $4 \mathrm{~b}$, entropy generation number increases with increasing viscosity parameter. This is particularly true in the region close to the annular pipe inner wall. Consequently, increasing rates of heat transfer and viscosity parameter decelerate the rate of entropy generation in the region close to the annular pipe wall. 
(a)

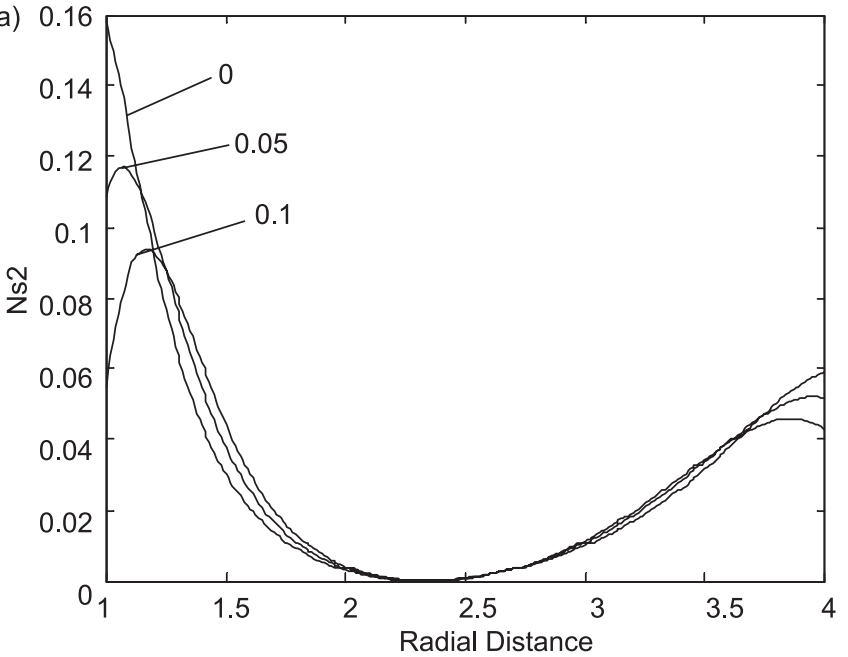

(b)

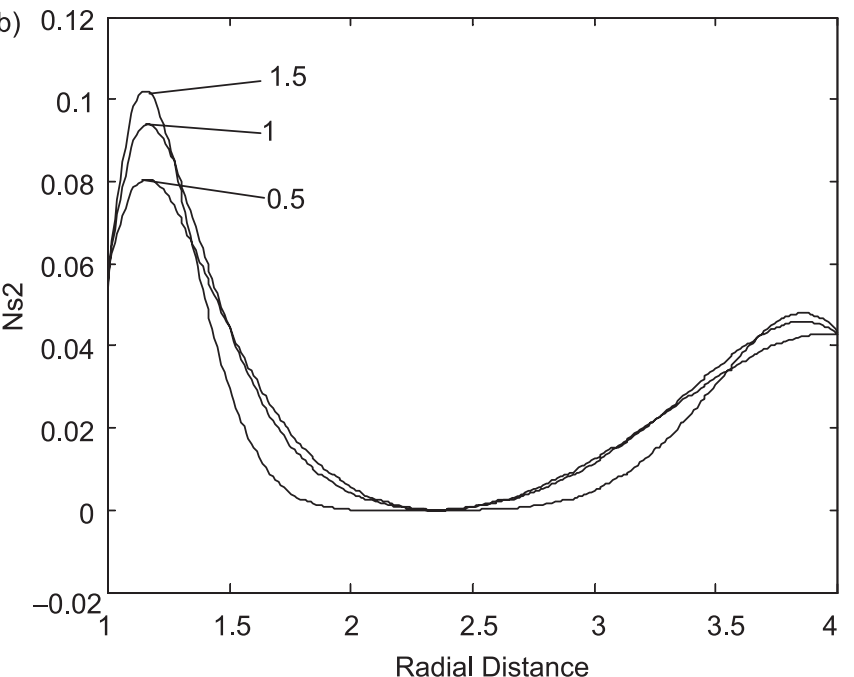

Figure 5. Entropy generation number due to fluid friction along the pipe radius for different non-Newtonian parameters

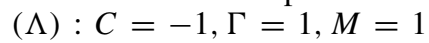
and $\theta_{0}=30$, and (b) different viscosity parameters: $C=$ $-1, \Lambda=0 \cdot 1, \Gamma=1$ and $\theta_{0}=$ 30.

Figures $5 \mathrm{a}$ and $\mathrm{b}$ show entropy generation number due to fluid friction for different nonNewtonian and viscosity parameters respectively. Entropy generation number attains high values in the region close to the annular pipe wall, particularly in the inner pipe wall region. Reducing non-Newtonian parameter increases the magnitude of entropy generation number in this region (figure 5a). This is because of fluid strain, which is high close to the annular pipe wall. Moreover, the maximum entropy generation number moves away from the pipe wall for a non-Newtonian parameter of less than 0.05 . In this case, the non-Newtonian parameter modifies the convective acceleration in the annular pipe resulting in the maximum rate of fluid strain moving away from the pipe wall. In the case of figure $5 \mathrm{~b}$, the maximum entropy generation number moves away from the pipe wall with increasing viscosity parameter. Increasing viscosity parameter reduces fluid viscosity and enhances fluid strain, particularly in the inner wall region of the annular pipe. This, in turn, modifies frictional losses in this region, resulting in maximum entropy generation rates occurring in this region. The intersecting of curves 

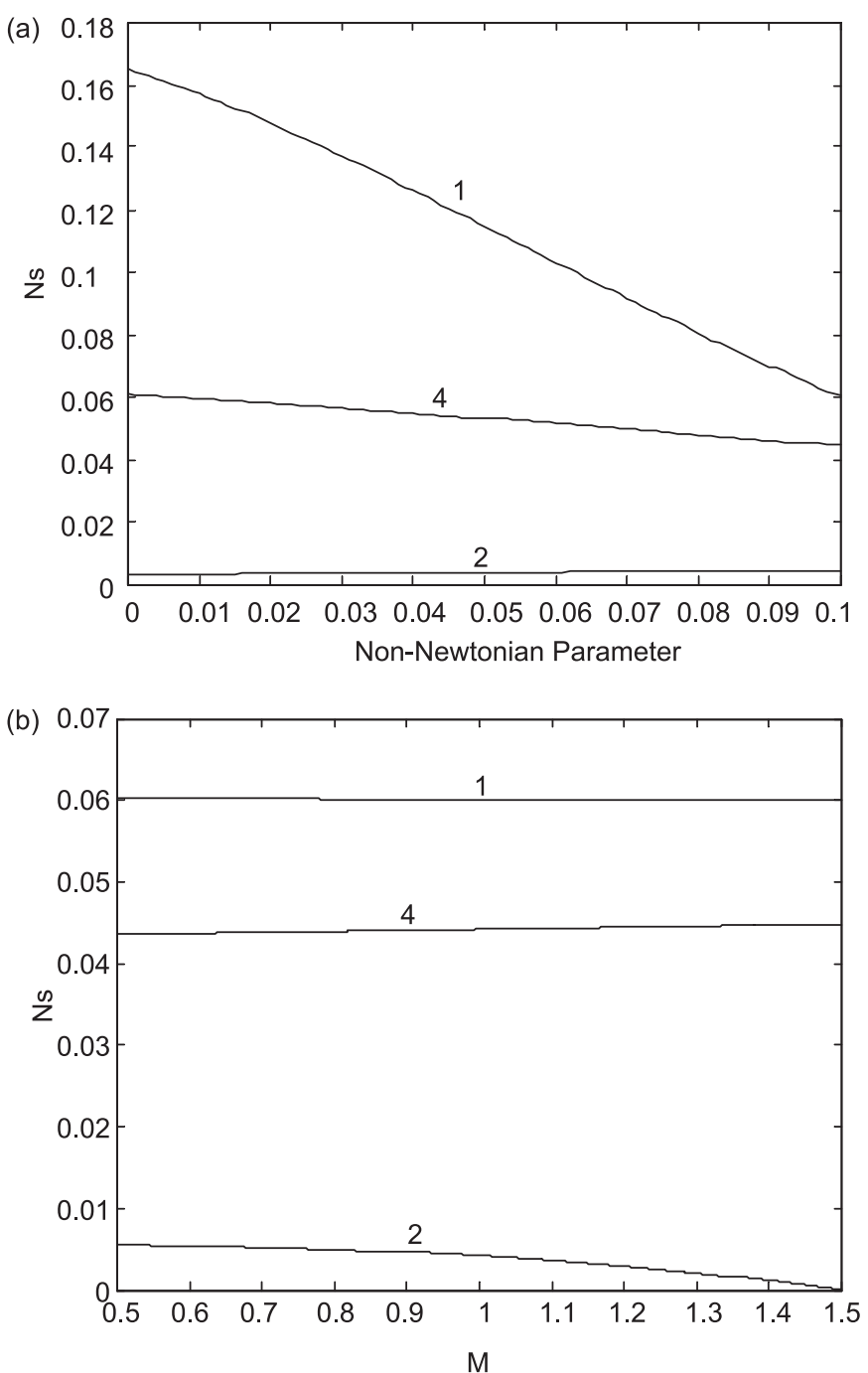

Figure 6. Entropy generation number due to heat transfer and fluid friction with (a) nonNewtonian parameter at different radial locations in the pipe: $C=-1, \Gamma=1, M=1$ and $\theta_{0}=30$, and (b) viscosity parameter at different radial locations in the pipe: $C=$ $-1, \Lambda=0 \cdot 1, \Gamma=1$ and $\theta_{0}=$ 30.

corresponding to different non-Newtonian parameters indicates that the rate of fluid strain changes in the inner and outer regions of the annular pipe walls, which in turn modifies the distribution of entropy generation number. A similar situation occurs for different viscosity parameters.

Figures $6 \mathrm{a}$ and $\mathrm{b}$ show the total entropy generation number at different locations in the annular pipe for different non-Newtonian and viscosity parameters respectively. Increasing non-Newtonian parameter lowers the total entropy generation number, particularly in the region close to the annular pipe inner wall. However, this variation is less significant for the central and outer region of the annular pipe. In the case of the viscosity parameter (figure $6 \mathrm{~b}$ ), the total entropy generation number increases with increasing viscosity parameter at all locations in the annular pipe, except at the radial location corresponding to $r=2$ where the total entropy generation number reduces with increasing viscosity parameter. This is because of both heat transfer and fluid friction contribution on the entropy generation rate in this region 
(figures 3 and 4). However, the influence of non-Newtonian and viscosity parameters on the total entropy generation number is almost linear, i.e. the total entropy generation number decreases almost linearly with increasing non-Newtonian parameter and this is vice versa for the viscosity parameter. The linear behaviour of total entropy generation parameter with non-Newtonian parameter and viscosity parameter indicates that the irreversibility associated with the non-Newtonian flow system in the annular pipe is a linear function of these parameters, provided that the influence of non-Newtonian parameter on the total irreversibility is more significant than the effect of the viscosity parameter.

\section{Conclusions}

Non-Newtonian fluid flow in an annular pipe is considered and the influence of non-Newtonian parameter and viscosity parameter on entropy generation number due to fluid friction and heat transfer is examined. Reynold's model is accommodated to account for temperaturedependent viscosity, while a third-grade fluid is employed to model the non-Newtonian fluid characteristics. It is found that reducing non-Newtonian parameter increases the maximum velocity magnitude as well as temperature in the annular pipe. However, a similar effect is observed for decreasing viscosity parameter. In this case, reducing non-Newtonian parameter and increasing viscosity parameter enhance the rate of fluid strain and temperature gradient in the region close to the annular pipe wall, which is more pronounced in the inner wall region of the annular pipe. Entropy generation number due to fluid friction and heat transfer behaves similar with varying non-Newtonian parameter, i.e. increasing non-Newtonian parameter suppresses the entropy generation rate in the annular pipe. However, increasing viscosity parameter enhances the entropy generation rates due to fluid friction and heat transfer in the annular pipe. The total entropy generation number varies almost linearly with non-Newtonian and viscosity parameters, provided that it decreases with increasing non-Newtonian parameter. This is vice-versa for the viscosity parameter, particularly in the region close to the inner wall of the annular pipe.

\section{List of symbols}

C dimensionless pressure gradient;

$C_{1} \quad$ dimensional pressure drop in the axial direction;

$c_{1}, c_{2} \quad$ parametric constants in velocity and temperature profiles;

$d_{1}, d_{2} \quad$ parametric constants in temperature profiles;

$h_{1}, h_{2} \quad$ parametric constants in velocity profiles;

$k$ thermal conductivity;

$k_{1,2 \ldots, 12}$ parametric constants in temperature profiles;

$M \quad$ exponential constant for Reynold's model viscosity;

$m \quad$ ordered viscosity parameter $(M / \in)$;

$m_{1}, m_{2} \quad$ parametric constants in temperature profiles;

Ns dimensionless entropy generation number;

$N s_{1} \quad$ dimensionless entropy generation number due to heat generation;

$N s_{2} \quad$ dimensionless entropy generation number due to viscous dissipation;

$R^{*} \quad$ difference of dimensional outer and inner radii;

$\bar{r}, \bar{r}_{i}, \bar{r}_{o}$ dimensional radius, dimensional radius of inner cylinder, and dimensional radius of outer cylinder respectively; 


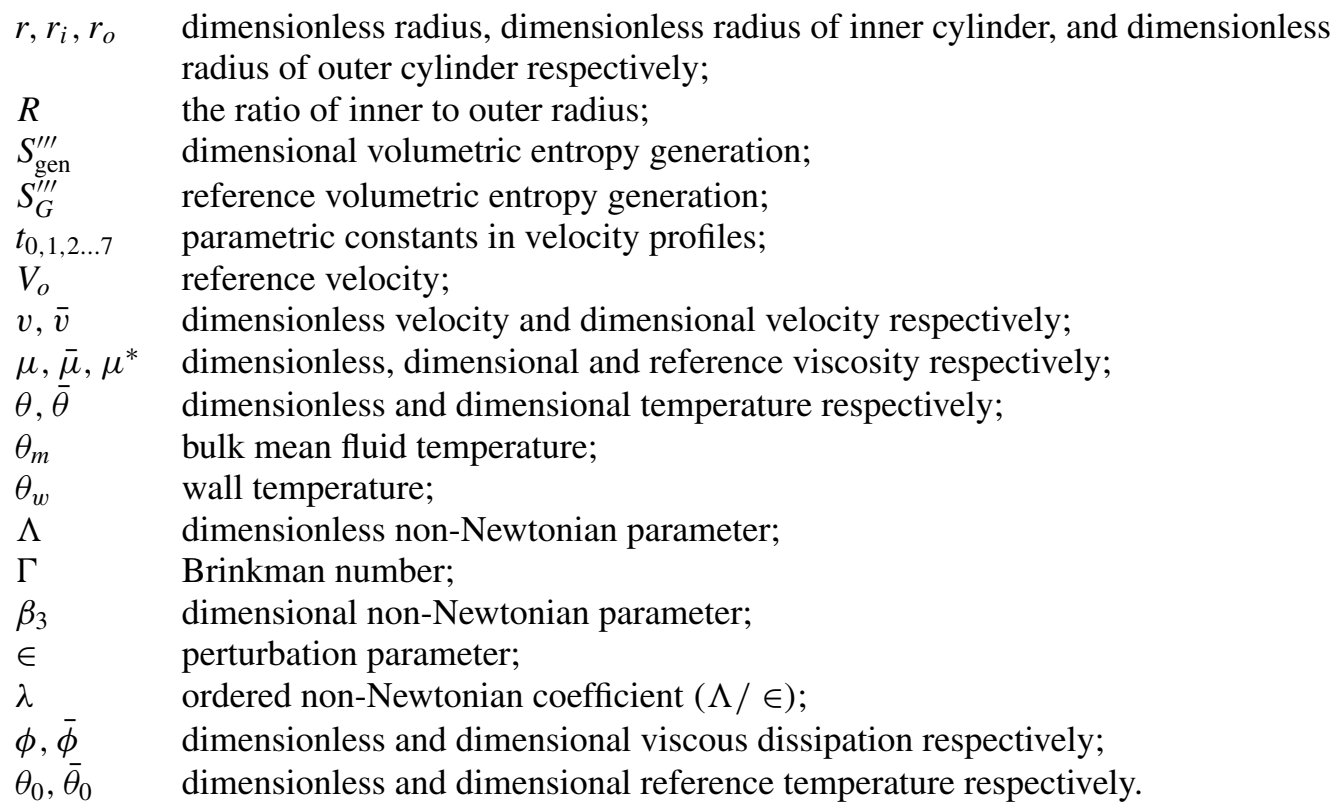

\section{References}

Bejan A 1995 Entropy generation minimization (New York: CRC Press)

Demirel Y, Kahraman R 2000 Thermodynamic analysis of convective heat transfer in an annular packed bed. Int. J. Heat Fluid Flow 21: 442-448

Hecht A M 1973 Theoretical non-Newtonian pipe-flow heat transfer. AIChE J. 19: 197-199

Massoudi M, Christie I 1995 Effect of variable viscosity and viscous dissipation on the flow of a third grade fluid in a pipe. Int. J. Nonlinear Mech. 30: 687-699

Peters G W M, Baaijens F P T 1997 Modelling of non-isothermal viscoelastic flows. J. Non-Newtonian Fluid Mech. 68: 205-224

Pinarbasi A, Ozalp C 2001 Effect of viscosity models on the stability of a non-Newtonian fluid in a channel with heat transfer. Int. Commun. Heat Mass Transfer 28: 369-378

Rajagopal K R, Sciubba E 1984 Pulsating Poiseulle flow of a non-Newtonian fluid. Math. Comput. Simulation 26: 276

Tanner R I 1966 Non-Newtonian fluid parameter estimation using conical flows. Ind. Eng. Chem. Fundam. 5: 55-59

Wang Y, Chukwu G A 1996 Unsteady axial laminar Couette flow of power-law fluids in a concentric annulus. Ind. Eng. Chem. Res. 35: 2039-2047

Yurusoy M 2004 Flow of a third grade fluid between concentric circular cylinders. Math. Comput. Appl. 9: 11-17

Yurusoy M, Pakdemirli M 2002 Approximate analytical solutions for the flow of a third-grade fluid in a pipe. Int. J. Nonlinear Mech. 37: 187-195

Zhang Z, Shi L 1999 Shannon entropy characteristics of two phase flow systems. J. Appl. Phys. 85: 7544-7551 